\title{
Influence of Cellulose Polymorphism on Tunable Mechanical and Barrier Properties of Chitosan/Oxidized Nanocellulose Bio- Composites
}

\author{
A.M. Adel ${ }^{1}$, A.M. El-Shafei ${ }^{2}$, M.T. Al-Shemy ${ }^{*}$, A.A. Ibrahim ${ }^{1}$ and A.M. Rabia ${ }^{3}$ \\ ${ }^{1}$ Cellulose and Paper Department and ${ }^{2}$ Textile Research Division, National Research \\ Center, Dokki, P.O. 12622, Giza and ${ }^{3}$ Department of Chemistry, Faculty of Science, \\ Ain Shams University, Cairo, Egypt.
}

OOD packaging based on biodegradable material attracts a lot of attention nowadays.
Chitosan (CS) is an available biopolymer that has been extensively studied as a potential
source for food packaging. However, its weak barrier for water vapor diffusion and mechanical
properties need to be improved. Oxidized nanocellulose (ONC) has been prepared from date
palm sheath fibers by the "green" ammonium persulfate (APS) hydrolysis. The particle size
distribution confirmed the success of ONC extraction. The CS/bio-composites were loaded with
$1,3,5,7$ and $10 \%$ ONC-I and II fractions. The FTIR spectroscopy analysis was carried out to
investigate the types of interaction exerts between these bio-composite components. The water
vapor permeability, water sorption isotherm and mechanical test of the neat CS film and CS/
bio-composite films were studied.Cellulose polymorphism was found to be an important factor
which could influence the bio-composite properties.

Keywords : Bio-composite, Food package, Polymorphism, Mechanical properties, Water vapor permeability, Water sorption isotherm.

\section{Introduction}

Nowadays, the eco-friendly food packaging attracts a global attention. Unlike petroleum derivatives food packages, safety, biodegradability, and low cost are the most relevant advantages of bio-composite package. In this regard the application of renewable and biodegradable biopolymers reinforced composite materials has been developed to get subsequent generation of sustainable and green materials in this field [1].

Among various available bio-composite materials, chitosan (CS) received considerable attention because of its unique properties such as; biodegradability, renewability and antibacterial effect. CS is derived from chitin, which is the second most abundant biopolymer on earth next to cellulose and is available from waste products in the shellfish industry [2]. However bio-composites with CS have several draw backs such as sensitivity to water, high affinity to water permeability and moderate mechanical properties. Many literatures which investigate the incorporation of another bio-polymer to the CS/ bio-composite matrix to enhance its barrier and mechanical properties were studied [3-5].
Cellulose which is the most abundant natural biopolymer exists in different polymorphic forms, namely, I, II, III and IV [6, 7]. The most studied polymorphic forms are cellulose I (CI) and cellulose II (CII). The difference between these two polymorphs lies in their crystallographic structure and chain orientation. While cellulose I has a parallel chain arrangement, cellulose II has an anti-parallelarrangement [8]. Mercerization treatment can irreversibly transform the crystal structure of cellulose I to that of cellulose II [9]. The production of cellulosic fibers in nano dimensions adds promising properties such as high mechanical characteristics and low density. Promising results were also obtained in nanocellulose applications to hybrid bio-composite materials, films, dispersions and foams, and in many other areas.

Recently, ammonium persulfate (APS) attracted attention owing to its properties being ideal for oxidizad nanocellulose (ONC) extraction [10]. This green oxidant is highly water soluble, inexpensive and commercially available. Therefore, APS as a strong oxidant has been used instead of acid hydrolysis to produce more homogeneous ONC. The use of APS results in the formation of highly carboxylated ONC 
directly without the need to undergo another step of 2,2,6,6-tetramethylpiperidine-1-oxyl radical (TEMPO) oxidation that is often needed for cellulose nanocrystals CNCs produced by acid hydrolysis [11].

The literature shows the importance of CNCs as their strength properties are much higher than those of several metallic and polymeric products available commercially. Lignocellulosic materials exhibit many advantages as compared to their synthetic counterparts, including their being toxicologically, and ecologically harmless $[1,12]$.

Date palm tree is one of the most widely distributed palms in the Middle East. Besides food-grade date production, large amounts of palm dates by-products end up as waste. The date palm leaf sheaths originate from the stem and surround it layer by layer. The palm fibers interweave in a network to form the leaf sheath fiber layers. As an abundant natural resource, the development and utilization of the date palm leaf sheath (DPS) fiber is being increasingly explored, e.g., in biocomposites, filters, elastic materials [13-16]. A literature showed indicated the production of CNCs from various sources of lignocellulosic materials as a base material [17-20], but the isolation of CNCs from DPS is still lacking. The authors are unaware of any previous publication concerning the appliance of CNCs from DPS isolated by using APS extraction in bio-composite application.

The main objective of the present study is to utilize ONCs from DPS isolated by APS extraction as a base material for production of bio-composite food packaging. Also, to investigate the effect of CI and CII polymorphism on mechanical and barrier properties of CS/bio-composites films. In addition, the utilization of the optimum ONC concentration, which could enhance the barrier and mechanical properties of $\mathrm{CS} /$ bio-composite for food packaging purpose.

\section{Experimental}

\section{Materials}

All chemicals used in this study were of analytical grade. CS with $90-95 \%$ degree of deacetylation and MW 400,000 was purchased from Oxford Laboratory, India. APS was supplied by Sigma Aldrich.

\section{a) Extraction of cellulose}

Cellulose was extracted from raw DPS fibers by the following successive steps. First, pulping with $18 \% \mathrm{NaOH}(\mathrm{w} / \mathrm{w})$ and liquor ratio (1:7) at $140^{\circ} \mathrm{C}$ for $1.5 \mathrm{hr}$ was carried out to remove waxy and/or any other non-cellulosic materials. The DPS pulp was bleached using the conventional three-stage method of sodium chlorite bleaching processes with $10 \%$ sodium chlorite (w/w), 1\% acetic acid (v/v) and $5 \%$ consistency at $70^{\circ} \mathrm{C}$ for $1 \mathrm{hr}$ for each step to remove the lignin completely and hemicellulose partially. For the complete removal of hemicellulose and conversion of cellulose I into cellulose II, the bleaching process was followed by mercerization treatment with $17.5 \% \mathrm{NaOH}(\mathrm{w} / \mathrm{v})$ for $3 \mathrm{hr}$ at $20{ }^{\circ} \mathrm{C}$. All stages of treatment were followed by washing with adequate distilled water till neutrality.

$$
\text { (ONC) }
$$

b) Preparation of oxidized nano-cellulose

For ONCs preparation the unbleached pulp and mercerized (CII) fibers, as obtained earlier, were treated by $(1-1.25 \mathrm{~mol} / \mathrm{L})$ APS solution, respectively and liquor ratio $(1: 100)$. The mixture was heated at $60^{\circ} \mathrm{C}$ for $(10-16 \mathrm{hr})$ in case of mercerized (CII) and pulp fibers, respectively under a continuous mechanical stirring to give a suspension of ONCs. The suspension was centrifuged at $12000 \mathrm{rpm}$ for $10 \mathrm{~min}$. The clear solution was decanted, and water was added to the ONC pellet, followed by successive centrifugation/washing cycles many times until the solution conductivity was $\approx 5 \mu \mathrm{S} \mathrm{cm}^{-1}(\mathrm{pH} 4)$. ONC suspensions were filtered in a sintered glass funnel No 1 before being lyophilized (Scheme 1).

\section{c) Bio-composite films formation}

The CS/ONC films were prepared by the solution casting method. CS solution was prepared by mechanically stirring $2 \% \mathrm{CS}(\mathrm{wt} / \mathrm{v})$ in $1 \%$ acetic acid solution $(\mathrm{v} / \mathrm{v})$ at $50^{\circ} \mathrm{C}$ for $3 \mathrm{hr}$. The non-dissolved matter and impurities were filtered through nylon filtration membrane. Bionanocomposite films were prepared by mixing CS solution with varying fractions of lyophilized ONCI and II from 0 to $10 \%$ (wt/wtCS). ONCI and II solution were prepared by an ultrasonic treatment (Falc Instruments, LSB2, $59 \mathrm{kHz}$ ) of lyophilized $\mathrm{ONC}$ fractions in $10 \mathrm{ml}$ distilled water for $10 \mathrm{~min}$ in an ice bath. Bio-nanocomposite blend was homogenized at $13500 \mathrm{rpm}$ for $5 \mathrm{~min}$. The homogenous mixtures were casted into PTFE plates after adding 30\% glycerol (wt/wtCS) as 
a plasticizer and stirring for $1 \mathrm{hr}$ at $50^{\circ} \mathrm{C}$. The casted films were subsequently dried at $35^{\circ} \mathrm{C}$ for $18 \mathrm{hr}$ in vented oven. The thickness of final films was around $70 \mu \mathrm{m}$. Films were conditioned at
$51 \% \mathrm{RH}$, using a desiccator containing saturated $\mathrm{Mg}\left(\mathrm{NO}_{3}\right)_{2}$ solution and $25{ }^{\circ} \mathrm{C}$ for 2 days before being tested.

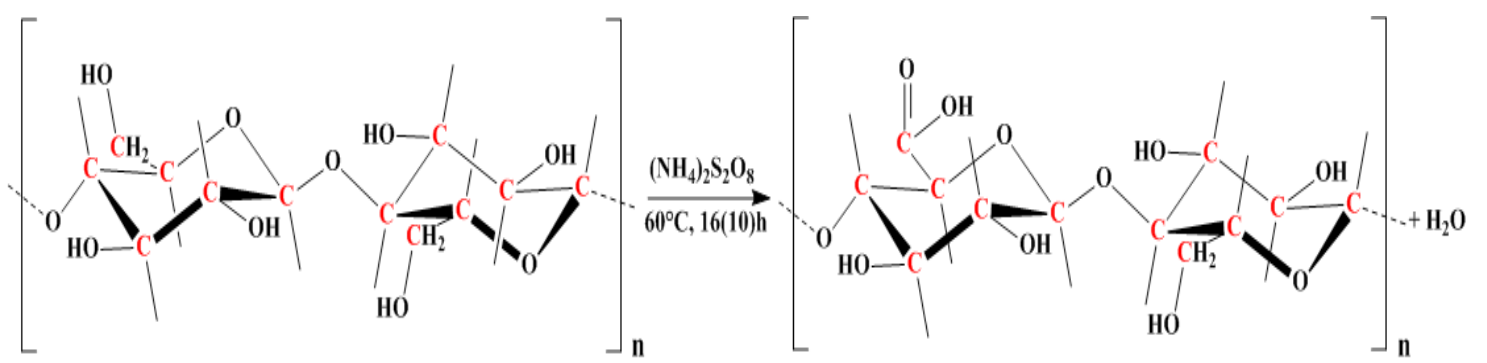

Scheme 1. Reaction byproduct of cellulose hydrolysis in aqueous APS.

Characterization of CS/bio-composite films

a) Particle size analysis

The particle size analysis of the prepared ONCs was carried out on a Malvern Nano Zetasizer (Malvern, NanoZS, UK) at $20 \pm 0.1^{\circ} \mathrm{C}$. A field of $40 \mathrm{~V}$ was applied across the nominal electrode spacing of $16 \mathrm{~mm}$. The samples were ultrasonicated in a sonication bath for 2 min prior to the measurements in order to increase particle dispersibility. The average values were calculated from at least twelve runs.

\section{b) Mechanical test}

The mechanical strength test was carried out using LLOYD LR 10k universal testing machine, England.The tensile specimens were cut in rectangular shapes with dimensions of $60 \mathrm{~mm}$ in length and $15 \mathrm{~mm}$ in width. The gauge length was fixed at $20 \mathrm{~mm}$ and the speed of the moving clamp was $10 \mathrm{~mm} / \mathrm{min}$. All tests were performed on a minimum of five samples and the reported results are average values.

c) Water vapor permeability measurement

Water vapor permeability (WVP) was determined according to ASTM E96-E80 [21]. Film specimens with approximately $5 \mathrm{~cm}$ diameter were mounted on the glass cups containing $1 \mathrm{~g}$ of anhydrous calcium chloride. Melted paraffin wax was used to fix the film specimen with the wide rim of the glass cup. They were weighed and then placed in desiccators containing saturated sodium chloride solution. The relative humidity of the chamber was kept at $75 \%$ and $25^{\circ} \mathrm{C}$ throughout the experiment. Weight gain of the cups covered with films was recorded daily for 5 days. The test was performed in triplicate.WVP was calculated by using the following equation:

$$
\mathrm{WVP}=\mathrm{W} x / \mathrm{t} A \Delta \mathrm{P}
$$

where $(\mathrm{W} / \mathrm{t})=$ the slope of the plot between weight loss and time, $x=$ the average thickness of the films, $\mathrm{A}=$ the permeation area, and $\Delta \mathrm{P}=$ the partial water vapor pressure difference of the atmosphere in the cup and saturated sodium chloride solution corresponding to $0-75 \% \mathrm{RH}$ (i.e., $2.385 \mathrm{kPa}$ ).

\section{d)Water sorption isotherm measurement}

Water sorption test of the prepared biocomposite was carried out in triplicate Film pieces $(20 \mathrm{X} 20 \mathrm{~mm} 2)$ were transferred to vacuum oven, dried at $50^{\circ} \mathrm{C}$ for $72 \mathrm{hr}$. Subsequently, film specimens were placed in desiccators containing $\mathrm{P}_{4} \mathrm{O}_{10}$ for 1 week at room temperature, and weighed on an analytical balance $(0.0001 \mathrm{~g})$. Then the film specimens were transferred to other activating desiccating chambers containing oversaturated salt solutions $\left(\mathrm{LiCl}_{3}, \mathrm{CaCl}, \mathrm{K}_{2} \mathrm{CO}_{3}, \mathrm{Mg}\left(\mathrm{NO}_{3}\right)_{2}\right.$, $\mathrm{NaCl}$ and $\mathrm{K}_{2} \mathrm{SO}_{4}$ ) at $25^{\circ} \mathrm{C}$ to maintain the relative humidity at a constant level $(\mathrm{RH}=11,31,43,51$, 75 and $97 \%$, respectively). Samples were weighed periodically until constant weight is reached. The equilibrium moisture sorption (EMS) isotherms were determined by the gravimetric method according to the following equation [22].

$$
\mathrm{EMS}=100 \mathrm{X} \mathrm{M} / \mathrm{M}^{\prime}
$$

where $M$ is the mass of water absorbed at equilibrium and $\mathrm{M}^{\prime}$ is the dry weight of the material. The moisture sorption profile represented by the plot of EMS versus water activity of each saturated salt solution was then evaluated.The monolayer sorption is calculated according to BET theory by the following equation. 


$$
\mathrm{M}=\mathrm{M}_{0} \mathrm{C} \mathrm{a}_{\mathrm{w}} /\left[\left(1-\mathrm{a}_{\mathrm{w}}\right)\left(1+(\mathrm{C}-1) \mathrm{a}_{\mathrm{w}}\right)\right]
$$

which can be simplified to:

aw $/\left(1-a_{w}\right) M=\left(1 / M_{0} C\right)+\left[(C-1) a_{w} / M_{0} C\right]$

where $\mathrm{a}_{\mathrm{w}}$ is the water activity, $\mathrm{C}$ is energy constant and $\mathrm{M}_{0}$ is the monolayer moisture content [23].

\section{e) FTIR spectroscopy analysis}

FT-IR spectra of the neat CS film and CS/ ONC bio-composite were recorded on JASCO FTIR 6100 spectrometer (Tokyo, Japan) directly. Whereas, the FT-IR spectra of basic CS powder and lyophilized $\mathrm{ONC}$ were analyzed as $\mathrm{KBr}$ pellets ( $1 \%$ cellulose in anhydrous $\mathrm{KBr})$. The absorbance measurements were carried out within the range of 4000-400 $\mathrm{cm}^{-1}$, with 60 scans and a resolution of $4 \mathrm{~cm}^{-1}$.

\section{Results and Discussion}

Particle size analysis of $O N C$

The particle size distributions of ONCs, prepared by the APS hydrolysis of CI and CII, display particles with sizes smaller than 100 $\mathrm{nm}$, i.e., nano-crystals were obtained. ONCI and ONCII had particles with sizes between 21.0 to $295.3 \mathrm{~nm}$. Although some particles appear with the size larger than $100 \mathrm{~nm}$, but those particles intensities were lower than $0.01 \%$ and the particles smaller than $100 \mathrm{~nm}$ distinguished by the highest intensities. In terms of particle sizes distribution, the specimens of ONCII were smaller than ONCI. The particle size distributions of ONCs display particles with sizes smaller than $100 \mathrm{~nm}$. ONCI and ONCII had particles with sizes between 21.0 to $105.7 \mathrm{~nm}$. ONCI had a relatively larger average particle size of $37.4 \mathrm{~nm}$ and width $15.4 \mathrm{~nm}$. While the average particle size and width of ONCII is $35.5 \mathrm{~nm}$ and $12.7 \mathrm{~nm}$. In terms of particle sizes distribution, the specimens of ONCII were smaller than ONCI (Table 1). The mercerization treatment preceding the hydrolysis process appeared to be valuable in decreasing the particle sizes. This agrees well with what was found when the influence of the polymorphism of CI and CII on the formation of CNCs during sulfuric acid hydrolysis was investigated [24]. Although small particles were obtained, some agglomeration occurred. This could be due to the high hydrophilic nature of cellulose, causing the aggregation during its drying (i.e., hornification). This can be eliminated by the widely reported adjustment of the hydrophilic-hydrophobic balance and surface modeling $[25,26]$.

\section{Mechanical properties of CS/bio-composite loaded with ONC}

The tensile properties of the neat CS $(0 \%$ $\mathrm{ONC}$ ), and $\mathrm{CS} / \mathrm{ONC}$ bio-composite films were investigated by uniaxial tensiletests. The tensile strength (TS) at maximum load, the elongation at break (EB\%), the Young's modulus (YM), and the stiffness (S) of neat $\mathrm{CS}$ and CS/ONC biocomposite films are presented as functions of ONC contents in Fig. 1 and the values of these selected properties are presented in Table 2. The TS represents the maximum stress value applied to the material, the EB \% is defined as the strain to break of the material, the YM can be defined as the slope of the linear elastic deformation of the stress-strain curve, and the $\mathrm{S}$ is the energy needed to break the material, and can be calculated from the area under the stress-strain curve [27].

TABLE 1. Particle size distribution of ONCI and ONCII.

\begin{tabular}{|c|c|c|}
\hline \multirow{2}{*}{ Size (d.nm) } & ONCI & ONCII \\
\hline & \multicolumn{2}{|c|}{ No. $\%$} \\
\hline 21.0 & 2.03 & 3.821 \\
\hline 24.4 & 9.06 & 13.741 \\
\hline 28.2 & 18.11 & 21.71 \\
\hline 32.7 & 22.27 & 21.57 \\
\hline 37.8 & 19.73 & 16.39 \\
\hline 43.8 & 13.83 & 10.51 \\
\hline 50.8 & 8.07 & 6.01 \\
\hline 58.8 & 4.03 & 3.18 \\
\hline 68.1 & 1.75 & 1.59 \\
\hline 78.8 & 0.67 & 0.77 \\
\hline 91.3 & 0.23 & 0.36 \\
\hline 105.7 & 0.08 & 0.17 \\
\hline 122.4 & 0.03 & 0.08 \\
\hline 141.8 & 0.02 & 0.04 \\
\hline 164.2 & 0.02 & 0.02 \\
\hline 190.1 & 0.02 & 0.02 \\
\hline 220.2 & 0.02 & 0.01 \\
\hline 255.0 & 0.01 & 0.01 \\
\hline 295.3 & 0.01 & 0.00 \\
\hline
\end{tabular}

According to the results illustrated in Fig. 1, the tensile properties of neat CS film were affected by the addition of different ONC fractions. The neat CS film exhibits TS of $16.74 \mathrm{MPa}$, an $\mathrm{EB} \%$ of $50.81 \%$, a YM of $113.65 \mathrm{MPa}$, and a S of 7.67 $\mathrm{kN} / \mathrm{m}$. Crystallographic variations of ONCs have been investigated. The CS matrix was blended with carboxylated ONCI and II polymorphs in order to study the effect of crystallographic structure on 
TABLE 2. Tensile strength, elongation at break, Young's Modulus and Stiffness of CS/bio-composite films loaded with ONCI and ONCII fractions.

\begin{tabular}{|c|c|c|c|c|c|}
\hline & CS/ONC films & TS (MPa) & ЕВ \% & YM (MPa) & $S(k N / m)$ \\
\hline \multirow{5}{*}{ 苛 } & 0 & $16.74 \pm 1.9$ & $50.80 \pm 5.3$ & $113.65 \pm 12.6$ & $7.67 \pm 0.85$ \\
\hline & 1 & $18.40 \pm 3.0$ & $41.69 \pm 6.3$ & $117.48 \pm 25.4$ & $10.86 \pm 3.56$ \\
\hline & 3 & $19.07 \pm 2.9$ & $58.24 \pm 8.2$ & $112.82 \pm 33.4$ & $13.47 \pm 3.02$ \\
\hline & 5 & $24.13 \pm 4.0$ & $51.78 \pm 5.8$ & $238.24 \pm 40.1$ & $17.17 \pm 3.50$ \\
\hline & 7 & $31.70 \pm 4.3$ & $52.65 \pm 8.9$ & $413.28 \pm 50.8$ & $23.82 \pm 4.09$ \\
\hline \multirow{6}{*}{ 光 } & 10 & $26.27 \pm 4.5$ & $46.05 \pm 9.0$ & $437.46 \pm 60.2$ & $33.90 \pm 5.57$ \\
\hline & 1 & $14.51 \pm 1.9$ & $29.95 \pm 5.4$ & $178.71 \pm 25.3$ & $9.38 \pm 1.96$ \\
\hline & 3 & $16.26 \pm 1.8$ & $33.05 \pm 4.6$ & $164.45 \pm 32.7$ & $9.03 \pm 1.71$ \\
\hline & 5 & $18.66 \pm 2.4$ & $38.04 \pm 5.7$ & $269.14 \pm 45.9$ & $15.31 \pm 2.63$ \\
\hline & 7 & $24.93 \pm 2.5$ & $48.23 \pm 4.8$ & $273.26 \pm 44.6$ & $17.30 \pm 3.50$ \\
\hline & 10 & $16.22 \pm 2.7$ & $25.61 \pm 4.9$ & $311.23 \pm 34.4$ & $18.65 \pm 3.09$ \\
\hline
\end{tabular}

mechanical properties of CS films. The tensile properties of neat CS film were changed after blending with ONC. The CS/ONCI film exhibits a TS of $31.70 \mathrm{MPa}$ when $7 \%$ of ONCI were added. The EB\% of CS was not largely affected by the addition of 7\% ONCI. The YM and $\mathrm{S}$ increased with increasing the ONCI content and reached its maximum value of $(437 \mathrm{MPa}, 33.9 \mathrm{kN} / \mathrm{m}$, respectively) at $10 \%$ ONCI load. All of the biocomposites showed enhancements in the YM and $\mathrm{S}$ parameters. This could be an indication of homogeneous ONC dispersion through CS matrix.These new values observed for $\mathrm{CS} / \mathrm{ONC}$ film in comparison with neat CS suggest the establishment of bonds between the chains of both CS and ONC in the film, as deduced from the FTIR analysis. These bonds reinforce the network structure, thus modifying the tensile properties of the CS/ONC.These results are comparable to those reported by other authors working with $\mathrm{CS} /$ ONC bio-composites [24].

For CS/ONC bio-composite films, after the addition of $\mathrm{ONC}$ into the $\mathrm{CS}$ solution, a remarkable increase of tensile properties was clearly visible, especially in: (i) the tensile strength at maximum load, (ii) the modulus, and (iii) the stiffness. This could be due to the formation of a more bonded network that was generated by the addition of ONC within the CS matrix. This network was formed throughout the strong electrostatic interactions and hydrogen bonding that occurred between the ONC and CS polymers (Scheme 2).

With regard to CS/ONCII blend, the TS and $\mathrm{YM}$ of the CS/ONCII bio-composite increased only by $48.9 \%$ from 16.74 to $24.93 \mathrm{MPa}$, and by $140.44 \%$ from 113.65 to $273.26 \mathrm{MPa}$ when $7 \%$
ONCII (wt/wtCS) was added. Such improvements confirm that $\mathrm{CS} / \mathrm{ONC}$ bio-composite films are mechanically strong materials. Furthermore, it should be noted that in comparison to the uncharged neat $\mathrm{CS}$, the $\mathrm{CS} / \mathrm{ONC}$ films not only have a higher strength and modulus but also a higher $\mathrm{S}$ that reaches a maximum value of 18.65 $\mathrm{kN} / \mathrm{m}$ for the CS/ONCII bio-composite film.

The EB\% of CS was not largely affected by the addition of $7 \%$ ONCI and ONCII, whereas a decrease from $50.8 \%$ for neat CS film to $25.61 \%$ for $\mathrm{CS} / \mathrm{ONCII}$ at $10 \%$ ONCII loading occurred. The gradual increase of the $\mathrm{S}$ and the slight decrease of the $\mathrm{EB} \%$ determined for the $\mathrm{CS} / \mathrm{ONCI}$ and $\mathrm{CS} / \mathrm{ONCII}$ bio-composite films at $7 \%$ ONCs contents, confirm that these films are mechanically ductile materials. Apparently, the large surface area and very high YM of ONCI than ONCII are responsible for the significant reinforcement impacton mechanical properties of the CS polymer. In addition, homogeneous dispersion of ONC along with favorable interfacial interactions between $\mathrm{ONC}$ and the CS matrix is essential to achieve improvement in the final tensile properties of the asprepared biocomposite films. These ideal conditions result in mechanically strong and ductile bio-composite films [27].

As shown in Fig. 1, films made from the 7\% ONC had the highest TS for both ONCI and ONCII blend. Bio-composite films made from ONCI had higher TS than that made from ONCII. The difference in ductility of ONCI and ONCII is probably due to the difference in their particle morphology and/ or hydrogen bonding capability [24]. The elongation at break and the elongation 

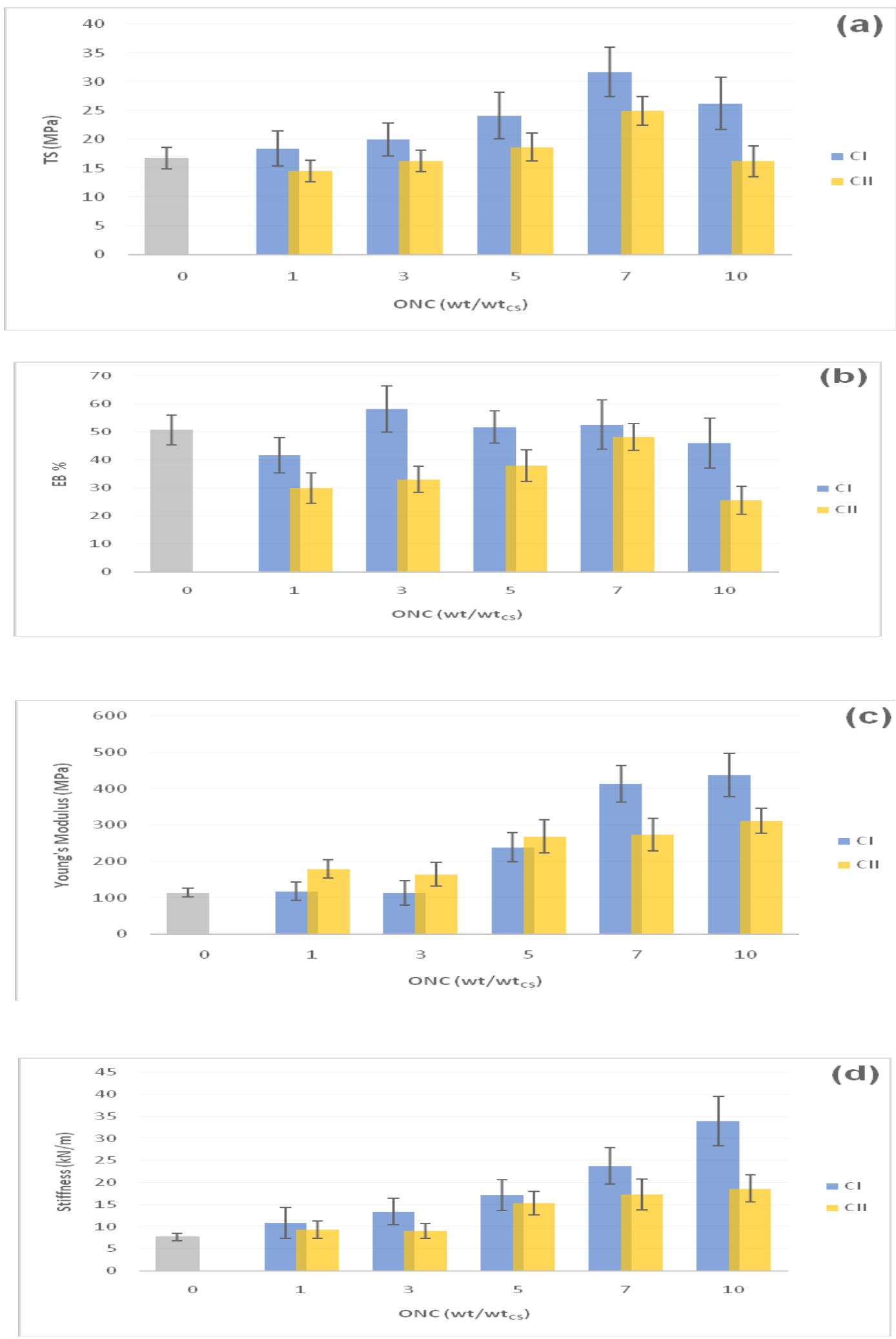

Fig. 1. Tensile strength (a), elongation at break (b), Young's Modulus (c) and stiffness (d) of CS biocomposite with varied fractions of ONCI and ONCII.

Egypt.J.Chem. Vol. 60, No.4 (2017) 


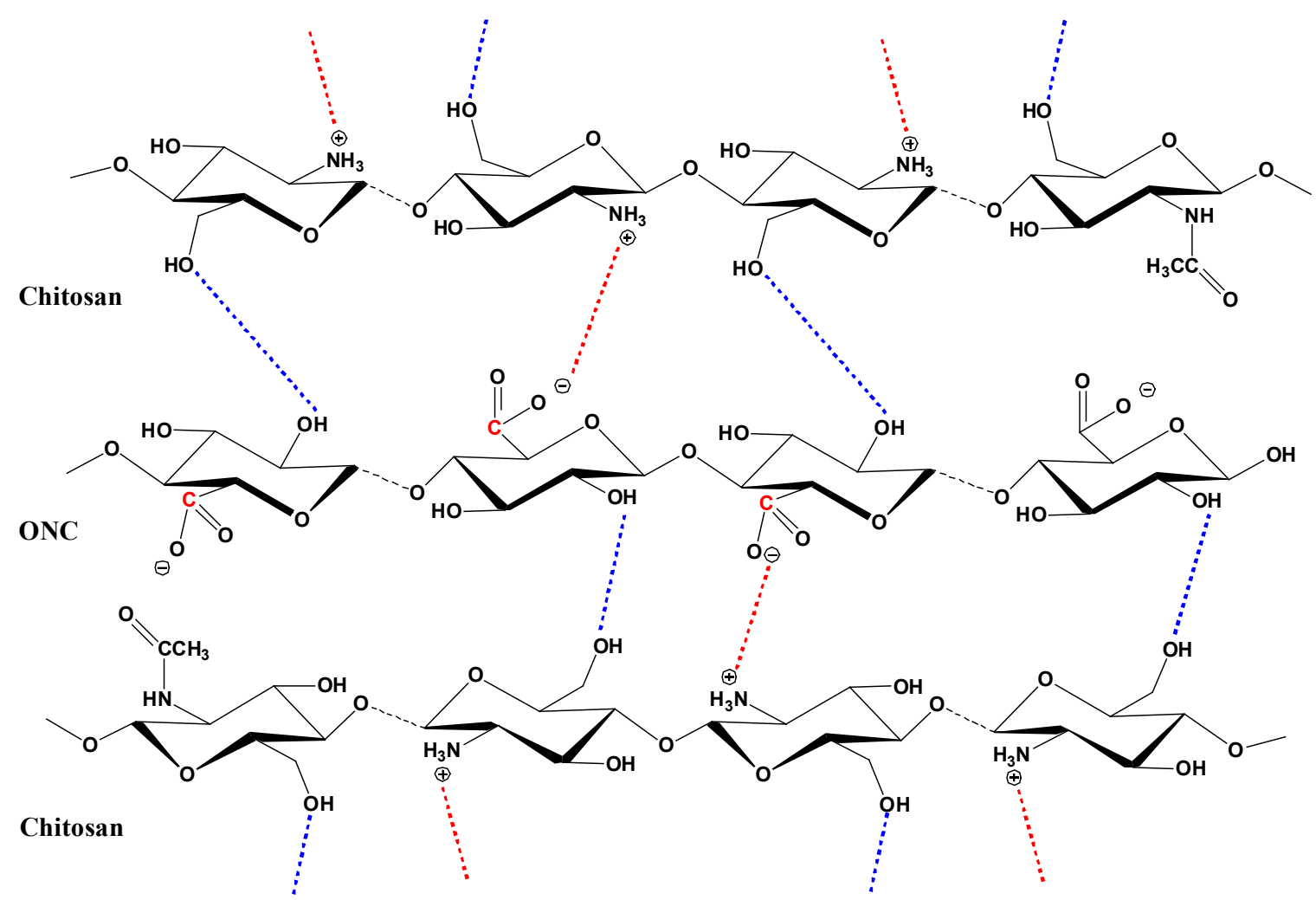

Scheme 2. Representation of electrostatic interactions and hydrogen bonds in the bio-composites matrix at low

pH.

at ultimate load are relatively the same. The $\mathrm{EB} \%$ of CS/ONCI bio-composite films was not affected so much by ONCI load, while EB\% of CS/ONCII bio-composite films decreased from $50 \%$ of neat CS films to $25 \%$ with 10 ONCs load. Similar results were observed previously when bio-composite films consisting of CS and nanocellulose were prepared by sulfuric acid hydrolysis [24], and when alginate biocomposites films were loaded with bagasse and rice straw cellulose nanofibers [27]. However, the $\mathrm{EB} \%$ of the bio-composites was found to be decreased as the nanocellulose content increased, the incorporation of $\mathrm{ONC}$ restricts the motion of the CS matrix in terms of the strong interactions between the fillers and biopolymer matrix. In our work a plasticizer (glycerol) was added in order to overcome this brittleness of films caused by intermolecular forces.

Mechanical strength investigation suggested that the tensile properties of the prepared biocomposites were directly related to the cellulose polymorphism. The results demonstrated that the bio-composites containing ONCII were characterized by reduced mechanical parameters. Similar results were found when alkalized wood pulp was used in the polypropylene composites formation [28]. Conversely, some authors confirmed the positive role of mercerization in the improvement of the mechanical properties of the composites [29, 30].

The ONC polymorph shapes were linked with an important factor: the aspect ratio, which is the ratio of the length to the width of the nanocellulose fiber. The importance of aspect ratio is due to its establishment of both the anisotropic phase formation and the reinforcing properties [27]. A relatively large aspect ratio is responsible for high reinforcing properties and, therefore, has been studied extensively in the literature. In our research, a greater reinforcing effect was obtained for bio-composites of ONCI with the higher aspect ratios [27].

\section{Water vapor permeability}

Water vapor permeability is one of the most important properties for food packaging applications due to its relation with food spoilage reactions. Figure 2 shows the water vapor permeability as a function of ONC concentration for the neat and $\mathrm{CS} / \mathrm{ONC}$ bio-composite films. As 
can be seen, with the increase of ONCI content, the values of WVP decreased significantly from $2.51 \times 10^{-3} \mathrm{~g} / \mathrm{m} . \mathrm{s} . \mathrm{kPa}$ for the neat CS film to $1.41 \times 10^{-3} \mathrm{~g} / \mathrm{m} . \mathrm{s} . \mathrm{kPa}$ for the CS-ONC film with $7 \%$ ONCI loading. Further increase of ONCI concentration over $7 \%$, didn't reduce WVP significantly. The presence of ONCs is thought to enhance the tortuosity in the CSfilms leading to slower water vapor diffusion processes and therefore, to a lower permeability [31]. WVP of CS/ONCII bio-composite film decreased significantly by loadingt he neat CS film with $3 \%$ ONC to $1.74 \times 10^{-3} \mathrm{~g} / \mathrm{m} . \mathrm{s} . \mathrm{kPa}$. That is comparable to the WVP of CS/ONCI-5 $\left(1.72 \times 10^{-3} \mathrm{~g} / \mathrm{m} \mathrm{s} \mathrm{Pa}\right)$. However, the WVP increased with higher ONCII loading. This might be due to the higher fractions of the available hydroxyl groups from anti parallel arrangement of CII polymorph which in turnmay lead to the aggregation of ONC particles at higher loading (7\%) and as a consequence forming voids in the composite matrix. These results are in accordance with previous findings [32, 33].

\section{Water sorption isotherm}

The knowledge of the sorption isotherm is important for predicting stability and quality changes during the packaging of food products. Since the moisture sorption isotherm allows the characterization of the water absorption property of bio-composite under study. Experimental data for moisture sorption at $25{ }^{\circ} \mathrm{C}$ for $\mathrm{CS}$ biocomposite loaded with different $\mathrm{ONC}$ fractions

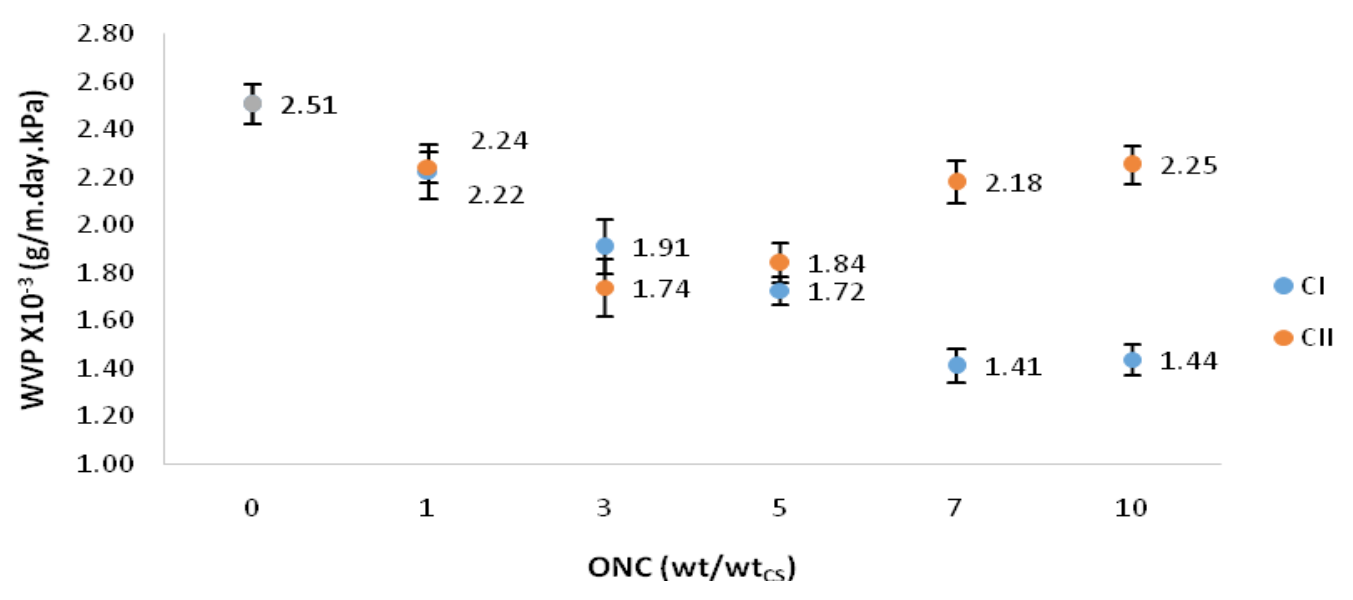

Fig. 2. Effect of ONCI and ONCII content on WVP of CS/ONCI and CS/ONCII bio-composite films.

and two ONC polymorphs are shown in Fig. 3. As we can expect, the sorption isotherm is sigmoid shaped curve of type II.

The equilibrium moisture content of the neat $\mathrm{CS}$ film is higher than $\mathrm{CS} / \mathrm{ONC}$ bio-composite films, showing a significant difference $(\mathrm{p}<0.05)$ between those films at the whole $\mathrm{a}_{\mathrm{w}}$ range. This difference is clearly apparent when CS/biocomposite is loaded with ONCI. The uniform dispersions of ONC within CS matrix suggesting the creation of an interlocking network structure within the polymer blend matrix which is capable of reducing water absorption by bio-composite films when exposed to moisture.

Among the wide variety of ways for describing sorption isotherms, the BET theory is the most successful and widely used in the food industry and food package due to its simplicity and more easily interpretable results with minimum data input. The BET theory utilizes the assumption that vander Waals forces account for sorption of water molecules on product surfaces. The BET monolayer value can be defined as the optimal water content for stability of materials at low water activity $(\geq 0.5)$ [23]. From Table 3 it is clear that unloaded CS/bio-composite has the highest amount of adsorbed monolayer water. Also, the amount of $\mathrm{M}_{0}$ decreased with increasing the percentage of ONC and after a certain concentration, it increased again. On the other hand, CS/bio-composite loaded with ONCI show lower $\mathrm{M}_{0}$ than those loaded with ONCII which is in agreement with our previous finding with WVP.

FTIR spectral analysis of CS/bio-composites with oxidized ONCI and ONCII

To gain insight into the mechanism of interaction between CS macromolecules and ONCs, the FTIR spectra of CS powder, lyophilized ONCs, neat CS 
film and CS/bio-composite films loaded with (0 to $10 \%)$ ONCI and ONCII were recorded. From IR profiles of ONCI and ONCII (Fig. 4), the broad

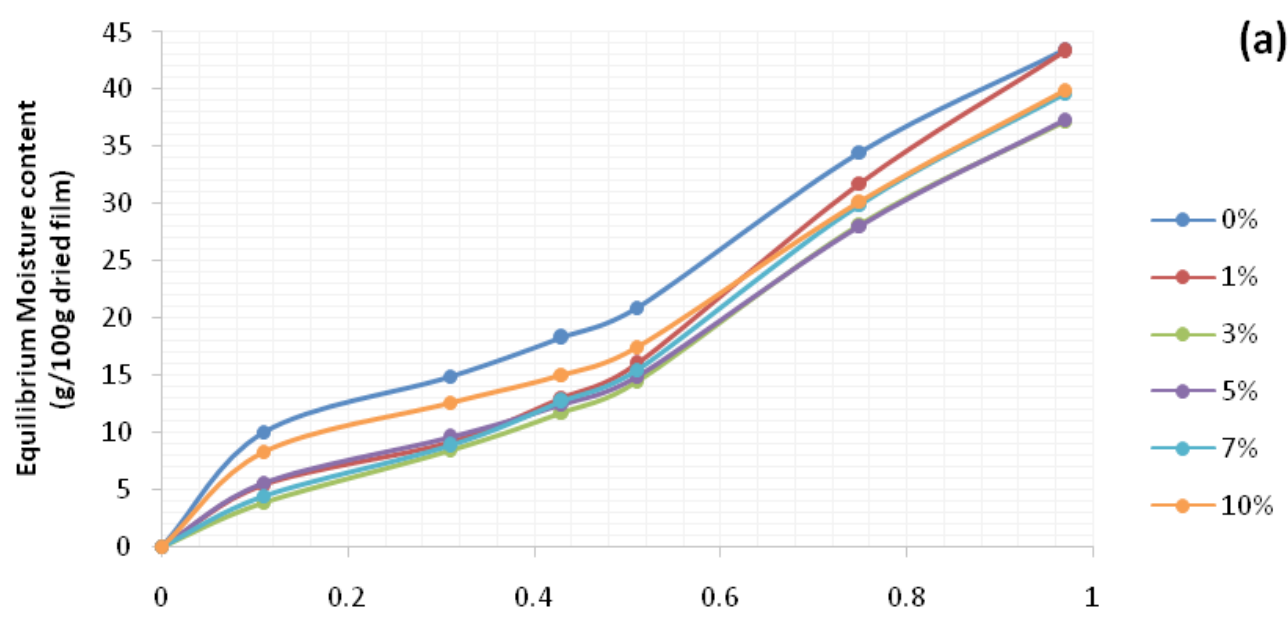

$a_{w}$

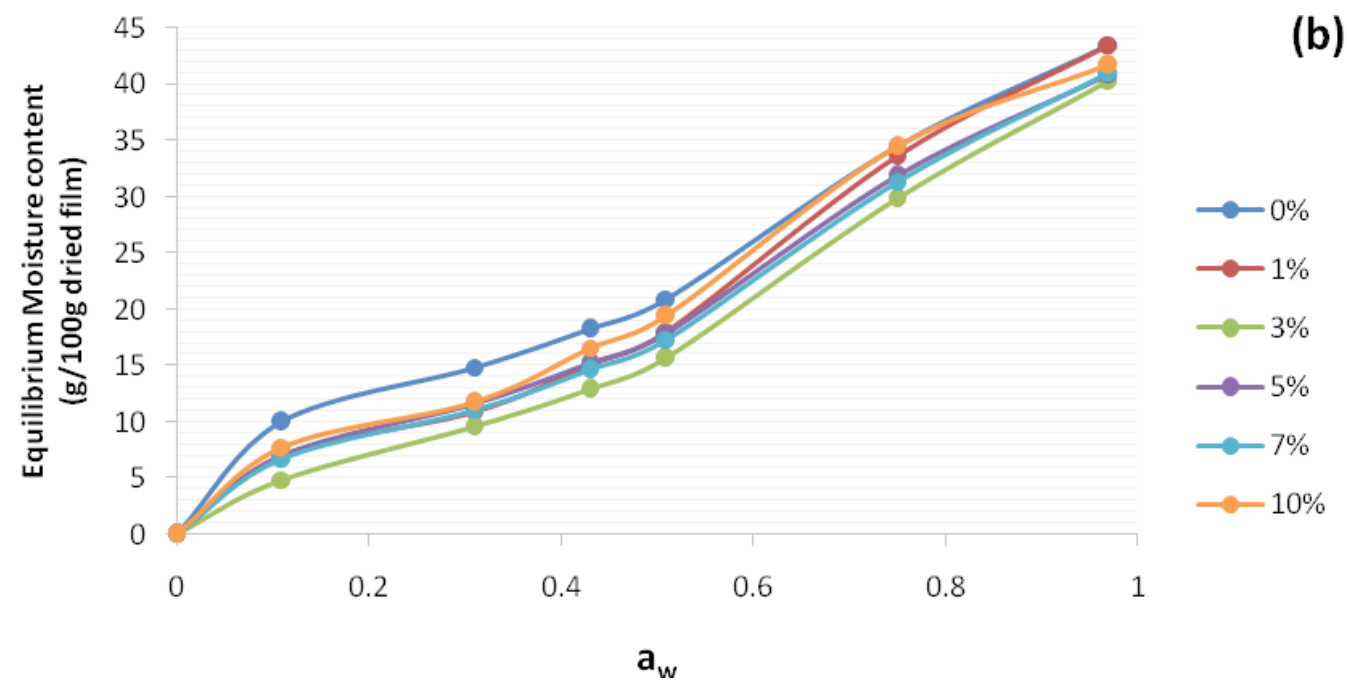

Fig. 3. Water sorption isotherm of CS bio-composite loaded with different fractions of (a) ONCI and (b) ONCII.

TABLE 3. Estimated parameters of BET model of CS/bio-composite loaded with different fractions of ONCI and ONCII.

\begin{tabular}{|c|c|c|c|c|}
\hline \multirow{2}{*}{ ONC content (wt/wt $\left.t_{C S}\right)$} & \multicolumn{2}{|c|}{ ONCI } & \multicolumn{2}{|c|}{ ONCII } \\
\hline & C & $\mathbf{M}_{0}$ & C & $\mathbf{M}_{0}$ \\
\hline 0 & 137.71 & 10.37 & 137.71 & 10.37 \\
\hline 1 & 24.96 & 7.70 & 35.59 & 8.78 \\
\hline 3 & 15.97 & 7.12 & 37.68 & 9.48 \\
\hline 5 & 37.16 & 7.27 & 42.04 & 8.81 \\
\hline 7 & 17.85 & 7.57 & 39.23 & 8.50 \\
\hline 10 & 145.00 & 8.62 & 37.68 & 9.48 \\
\hline
\end{tabular}

Egypt.J.Chem. Vol. 60, No.4 (2017) 
vibrations, and peaks at around $1650 \mathrm{~cm}^{-1}$ are due to the deformation vibration of water molecules. Absorption bands in the $1500-800 \mathrm{~cm}^{-1}$ spectral region, attributed to the $\mathrm{C}-\mathrm{H}, \mathrm{O}-\mathrm{H}, \mathrm{C}=\mathrm{O}$ and $\mathrm{C}-$ $\mathrm{O}-\mathrm{C}$ vibration on the glucosidic ring, represent the fingerprint of cellulose [34, 35].

From basic CS powder spectrum (Fig. 4), the broad bands in the region between 3650 and $3000 \mathrm{~cm}^{-1}$ is due to $\mathrm{NH}$ stretching vibrations. The peaks at 2902,1670 and $1597 \mathrm{~cm}^{-1}$ are associated with $\mathrm{C}-\mathrm{H}$ stretching, $\mathrm{C}-\mathrm{O}$ stretching of the acetyl group (amide $\mathrm{I}$ ) and $\mathrm{N}-\mathrm{H}$ bending and stretching (amide II), correspondingly. A weaker amino characteristic peak at $1256 \mathrm{~cm}^{-1}$ is associated with $\mathrm{O}-\mathrm{H}$ bending vibration and the peak at $1066 \mathrm{~cm}^{-1}$ is assigned to $\mathrm{C}-\mathrm{O}$ stretching. The absorption band at $1155 \mathrm{~cm}^{-1}$ and $897 \mathrm{~cm}^{-1}$ are due to the saccharide structure [3].

The neat CS film shows the characteristic peaks of basic CS powder (Fig. 4). The broad band at $3561 \mathrm{~cm}^{-1}$ is attributed to amine NH symmetric vibration, while $2985 \mathrm{~cm}^{-1}$ peak is assigned to $\mathrm{C}-\mathrm{H}$ vibration. The absorption bands at 1560 and $1334 \mathrm{~cm}^{-1}$ are assigned to the stretching vibration of amino group of $\mathrm{CS}$ and $\mathrm{C}-\mathrm{H}$ vibration, consequently. The peaks at 1170 and $896 \mathrm{~cm}^{-1}$ are corresponding to saccharide structure of CS. The broad peak at 1129 indicates $\mathrm{C}-\mathrm{O}$ stretching vibration $[36,37]$.

Figures 5 and 6 showed the differences in the FTIR spectra of CS/ONCI and CS/ONCII

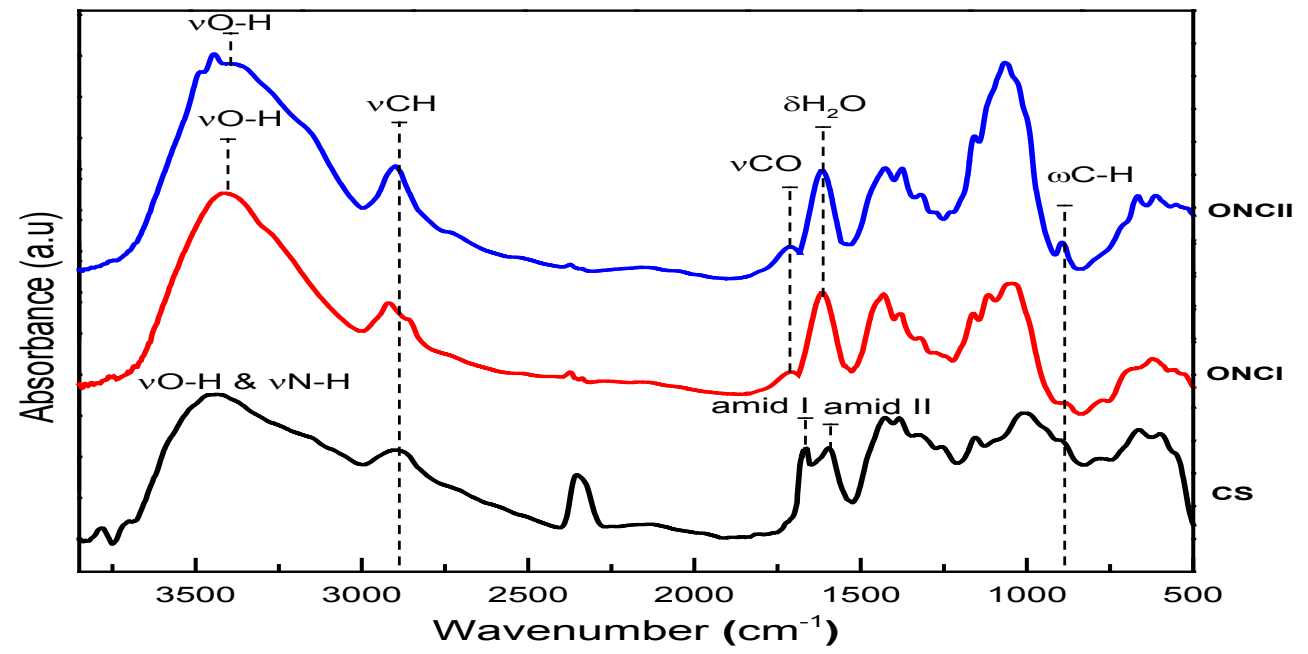

Fig.4 . FTIR spectra of basic CS powder, ONCI and ONCIIpowders.

bio-composites. All films showed characteristic bands of neat CS film. Typical changes in both shape and intensity of the main peaks were observed in the FTIR spectra of bio-composite films reinforced with ONCs. The absorption band at $3750-3000 \mathrm{~cm}^{-1}$ is due to the overlapped symmetric stretching vibration of $\mathrm{NH}_{2}$ and $-\mathrm{OH}$ groups from $\mathrm{CS}$ and $\mathrm{ONC}$, respectively. The absorption band at $3561 \mathrm{~cm}^{-1}$ of neat CS film shifted to lower wavelength, increased in intensity and became broader upon ONC blending. This can be ascribed to the possible hydrogen bonding network formed between the ONCs-OH and CS$\mathrm{NH}_{2}$ available groups [38]. The absorption peak at around $2885 \mathrm{~cm}^{-1}$ is due to $\mathrm{C}-\mathrm{H}$ stretching. As seen, the absorption peak of amide $\mathrm{I}$ (due to $-\mathrm{C}=\mathrm{O}$ stretching of hydrogen bonded $\mathrm{C}=\mathrm{O}-\mathrm{NHCH}_{3}$ group) bands appears at around $1663 \mathrm{~cm}^{-1}$. The broadened left shoulder of amide I peak may be due to $\mathrm{C}=\mathrm{O}$ stretching band at $1750 \mathrm{~cm}^{-1}$ from the added ONCs. The amideII (due to $\mathrm{N}-\mathrm{H}$ bending of $\mathrm{NH}_{2}$ group) bands appear at round $1589 \mathrm{~cm}^{-1}$. The absorption peaks at 1326 and $1080 \mathrm{~cm}^{-1}$ are due to $\mathrm{N}-\mathrm{H}$ bending vibration of primary amides and $\mathrm{C}-\mathrm{O}-\mathrm{C}$ stretching, respectively [39]. It is interesting to underline that the peak at $1440 \mathrm{~cm}^{-1}$, which is sensitive to ONC crystallinity, intensity increased upon increasing $\mathrm{ONC}$ contents.

As mentioned earlier, the CS/ONCI and CS/ ONCII amid I band at $1665 \mathrm{~cm}^{-1}$, has a broad left shoulder at $1760 \mathrm{~cm}^{-1}$ due to an overlapping $\mathrm{C}=\mathrm{O}$ stretching vibration from oxidized ONCI and ONCII. The absorption intensity of crystallinesensitive band at $1440 \mathrm{~cm}^{-1}\left(\mathrm{CH}_{2}\right)$ was increased by embedding of ONCs, while amorphous peak at $898 \mathrm{~cm}^{-1}$ almost disappeared, this could be an indication of higher crystalline components when compared to the neat CS film. 


\section{Conclusion}

CS/bio-composite films were blended with ONCI and ONCII in the presence of glycerol (as plasticizer). The addition of ONC to the CS/biocomposite clearly modified its mechanical and barrier properties. The polymorphism of cellulose also has an influence on bio-composite properties. The loaded CS/ONC bio-composite films has higher YM and $\mathrm{S}$ and lower amount of absorbed water than that of neat CS film, suggesting that the ONCs were well dispersed within the CS matrix, acting as an interpenetrated network within

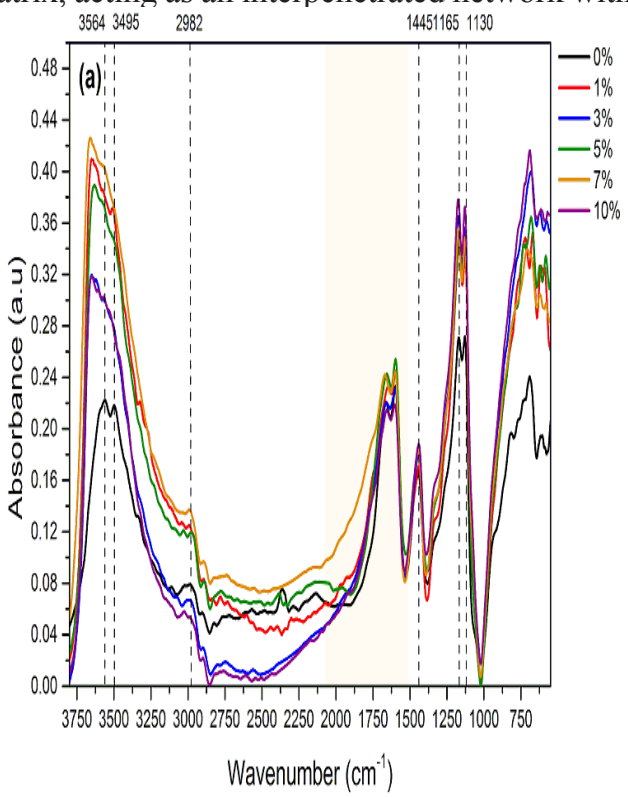

the polymer blend matrix, thus preventing the absorption of water by films when exposed to the moisture. CS/ONC bio-composite films loaded with ONCI show a higher mechanical and barrier properties than those loaded with ONCII as a result of the difference between them in the aspect ratio. Based on the obtained results of mechanical testing, WVP and water sorption isotherm, loading with $7 \%$ ONC was chosen as the optimum condition for $\mathrm{CS} /$ bio-composite film developing.

Fig.5. FTIR spectra of neat CS/bio-composite films loaded with different ONCI fractions (a); magnification of spectral range $2500-1550 \mathrm{~cm}^{-1}(\mathrm{~b})$.
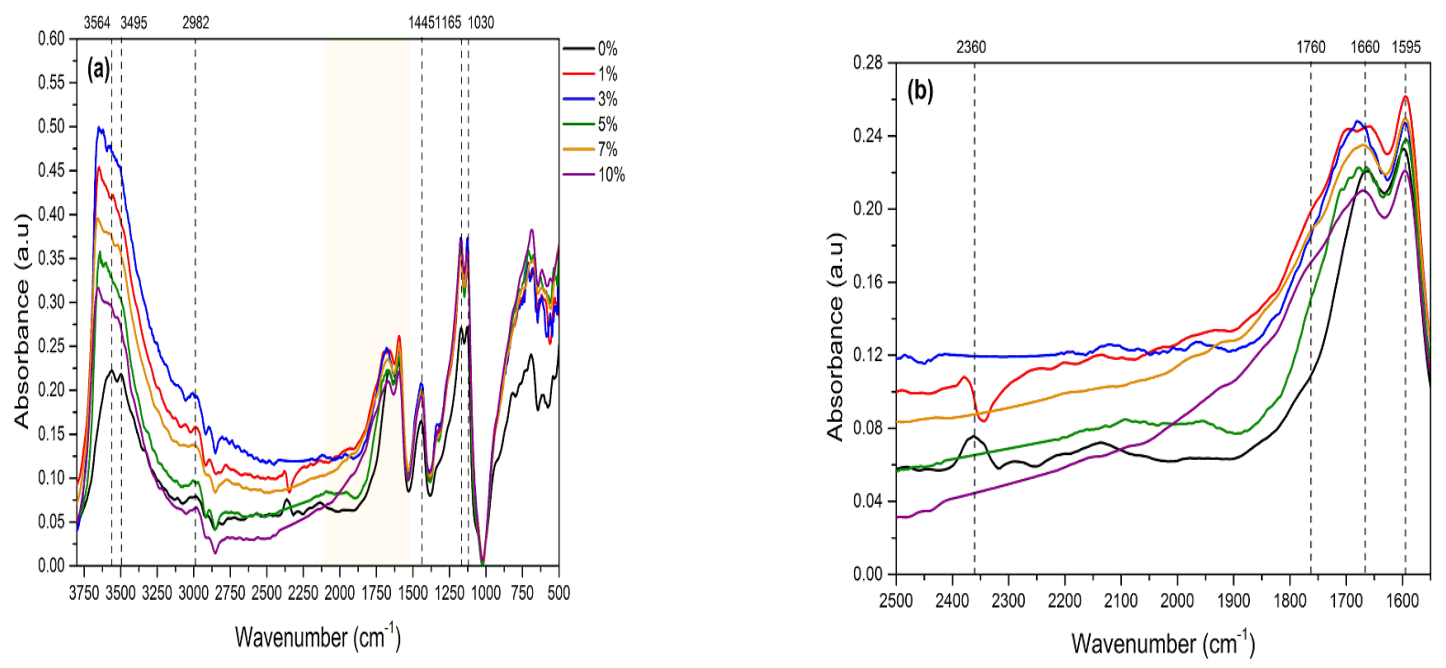

Fig. 6. FTIR spectra of neat CS/bio-composite films loaded with different ONCII fractions (a); magnification of spectral range $2500-1550 \mathrm{~cm}^{-1}(\mathrm{~b})$. 


\section{$\underline{\text { References }}$}

1. Fortunati, E., Rinaldi, S., Peltzer, M., Bloise, N., Visai, L., and Armentano, I. et al., Nanobiocomposite films with modified cellulose nanocrystals and synthesized silver nanoparticles. Carbohydrate Polymers,101,1122 (2014).

2. Dehnad, D., Mirzaei, H., Emam-Djomeh, Z., Jafari, SM. and Dadashi, S., Thermal and antimicrobial properties of chitosan-nanocellulose films for extending shelf life of ground meat. Carbohydrate Polymers, 109, 148 (2014).

3. Xu, Y.X., Kim, K.M., Hanna, M.A. and Nag, D., Chitosan-starch composite film: preparation and characterization. Industrial Crops and Products, 21,185 (2005).

4. Soni, B., Schilling, M.W., and Mahmoud, B., Transparent bionanocomposite films based on chitosan and TEMPO-oxidized cellulose nanofibers with enhanced mechanical and barrier properties. Carbohydrate Polymers,151,779 (2016).

5. HPS. A.K., Saurabh CK, A SA, Nurul Fazita MR, Syakir MI, Davoudpour Y, et al., A review on chitosancellulose blends and nanocellulose reinforced chitosan biocomposites: Properties and their applications. Carbohydrate Polymers, 150, 216 (2016).

6. Abdul Khalil, H.P., Davoudpour, Y., Islam, M.N., Mustapha, A., Sudesh, K., Dungani, R., and Jawaid, M., Production and modification of nanofibrillated cellulose using various mechanical processes: a review. Carbohydrate Polymers,99, 649 (2014).

7. Habibi, Y., Lucia, L.A. and Rojas, O.J., Cellulose nanocrystals: chemistry, self-assembly, and applications. Chemical Reviews,110, 3479 (2010).

8. Dinand, E., Vignon, M., Chanzy, H. and Heux, L., Mercerization of primary wall cellulose and its implication for the conversion of cellulose $\mathrm{I} \rightarrow$ cellulose II. Cellulose, 9, 7 (2002).

9. Ciolacu, D., Chiriac, A.I., Pastor, F.I. and Kokol, V., The influence of supramolecular structure of cellulose allomorphs on the interactions with cellulose-binding domain, CBD3b from Paenibacillus barcinonensis. Bioresource Technology, 157,14 (2014).

10. Zhang, K., Sun, P., Liu, H., Shang, S., Song, J. and Wang, D., Extraction and comparison of carboxylated cellulose nanocrystals from bleached sugarcane bagasse pulp using two different oxidation methods. Carbohydrate Polymers, 138, 237 (2016).

11. Leung, A.C., Hrapovic, S., Lam, E., Liu, Y., Male, K.B., Mahmoud, K.A., and et al., Characteristics and properties of carboxylated cellulose nanocrystals prepared from a novel one-step procedure. Small, 7, 302 (2011).

12. Vallejos, M.E., Peresin, M.S. and Rojas, O.J., All-Cellulose Composite Fibers Obtained by Electrospinning Dispersions of Cellulose Acetate and Cellulose Nanocrystals. Journal of Polymers and the Environment, 20,1075 (2012).

13. Alawar, A., Hamed, A.M. and Al-Kaabi, K., Characterization of treated date palm tree fiber as composite reinforcement. Composites Part B: Engineering, 40, 601 (2009).

14. Mahdavi, S., Kermanian, H. and Varshoei, A., Comparison of mechanical properties of date palm fiber-polyethylene composite. BioResources, 5, 2391 (2010).

15. Sahari, J., Sapuan, S., Ismarrubie, Z. and Rahman, M., Physical and chemical properties of different morphological parts of sugar palm fibres. Fibres and Textiles in Eastern Europe, 91, 21 (2012).

16. Cheng, L., Zhang, T., Guo, M. and Li, X., Structural characteristics and properties of windmill palm leaf sheath fiber. Wood Fiber Sci.,46, 270 (2014).

17. Yu, L., Lin, J., Tian, F., Li, X., Bian, F. and Wang, J., Cellulose nanofibrils generated from jute fibers with tunable polymorphs and crystallinity. Journal of Materials Chemistry A, 2, 6402 (2014).

18. Mandal, A., and Chakrabarty, D., Isolation of nanocellulose from waste sugarcane bagasse (SCB) and its characterization. Carbohydrate Polymers, 86, 1291 (2011).

19. Jonoobi, M., Khazaeian, A., Tahir, P.M., Azry, S.S. and Oksman, K., Characteristics of cellulose nanofibers isolated from rubberwood and empty fruit bunches of oil palm using chemo-mechanical process. Cellulose,18, 1085 (2011).

20. Neto, W.P.F., Silvério, H.A., Dantas, N.O. and Pasquini, D., Extraction and characterization of 
cellulose nanocrystals from agro-industrial residue - Soy hulls. Industrial Crops and Products,42, 480 (2013).

21. Standard A, Standard test methods for water vapor transmission of materials, Annual book of ASTM standards Designation E96-E80,730 (1989).

22. Zuo, L., Rhim, J.W. and Lee, J.H., Moisture Sorption and Thermodynamic Properties of Vacuum-Dried Capsosiphon fulvescens Powder. Preventive Nutrition and Food Science, 20, 215 (2015).

23. Adel, A.M. and El-shinnawy, N.A., Hypolipidemic applications of microcrystalline cellulose composite synthesized from different agricultural residues. International Journal of Biological Macromolecules, 51, 1091 (2012).

24. Borysiak, S. and Grząbka-Zasadzińska, A., Influence of the polymorphism of cellulose on the formation of nanocrystals and their application in chitosan/nanocellulose composites. Journal of Applied Polymer Science, 133, 42864 (2016).

25. Habibi, Y., Key advances in the chemical modification of nanocelluloses. Chemical Society Reviews, 43, 1519 (2014).

26. Mariano. M,, El Kissi, N. and Dufresne, A., Cellulose nanocrystals and related nanocomposites: Review of some properties and challenges. Journal of Polymer Science Part B: Polymer Physics, 52, 791 (2014).

27. Hassan, M., Kassem, N. and El-Sakhawy, M., Effect of Bagasse and Rice Straw Cellulose Nanofibers on Physico-Mechanical Properties of Alginate Nanocomposites Films. Egyptian Journal of Chemistry, 58, 299 (2015).

28. Borysiak, S., Fundamental studies on lignocellulose/ polypropylene composites: effects of wood treatment on the transcrystalline morphology and mechanical properties. Journal of Applied Polymer Science, 127,1309 (2013).

29. Pimenta, M.T.B., Carvalho, A.J.F., Vilaseca, F., Girones, J., López, J.P., Mutjé, P., and Curvelo, A.A., Soda-Treated Sisal / Polypropylene Composites. Journal of Polymers and the Environment, 16, 35 (2008).

30. Gassan, J. and Bledzki, A.K., Possibilities for improving the mechanical properties of jute/epoxy composites by alkali treatment of fibres. Composites Science and Technology,59, 1303 (1999).

31. Sanchez-Garcia, M., Gimenez, E. and Lagaron, J.,Morphology and barrier properties of nanobiocomposites of poly (3-hydroxybutyrate) and layered silicates. Journal of Applied Polymer Science, 108, 2787 (2008).

32. Reddy, J.P. and Rhim, J.W., Characterization of bionanocomposite films prepared with agar and paper-mulberry pulp nanocellulose. Carbohydrate Polymers,110, 480 (2014).

33. Mandal, A. and Chakrabarty, D., Characterization of nanocellulose reinforced semi-interpenetrating polymer network of poly (vinyl alcohol) \& polyacrylamide composite films. Carbohydrate Polymers,134, 240 (2015).

34. Adel, A.M., Abd El-Wahab, Z.H., Ibrahim, A.A. and Al-Shemy, M.T., Characterization of microcrystalline cellulose prepared from lignocellulosic materials. Part II: Physicochemical properties. Carbohydrate Polymers, 83, 676 (2011).

35. Shankar, S. and Rhim, J.W., Preparation of nanocellulose from micro-crystalline cellulose: The effect on the performance and properties of agar-based composite films. Carbohydrate Polymers,135, 18 (2016).

36. Afanas'Eva, N.V., Petrova, V.A., Vlasova, E.N., Gladchenko, S.V., Khayrullin, A.R., Volchek, B.Z. andBochek, A.M.,Molecular mobility of chitosan and its interaction with montmorillonite in composite films: Dielectric spectroscopy and FTIR studies. Polymer Science Series A, 55, 738 (2013).

37. El-Gendy, A.A., Abou-Yousef, H., Adel, A.M. and El-Shinnawy, N., Bio-based Hydrogel Formed by Gamma Irradiation. Egyptian Journal of Chemistry,59, 647 (2016).

38. Anicuta, S-G, Dobre, L., Stroescu, M. and Jipa, I., Fourier transform infrared (FTIR) spectroscopy for characterization of antimicrobial films containing chitosan. University Politehnica of Bucharest, Faculty Applied Chemistry and Material Science (2010).

39. Choo, K., Ching, Y., Chuah, C., Julai, S. and Liou, N-S., Preparation and Characterization of Polyvinyl

Egypt.J.Chem. Vol. 60, No.4 (2017) 
Alcohol-Chitosan Composite Films Reinforced with Cellulose Nanofiber. Materials, 9, 644 (2016).

(Received :4/6/2017;

accepted :9/7/2017)

\section{تأثير السليلوز متعدد الأثكال البللورية على الخصائص الميكانيكية وقابلية الحجز فى المثراكبات الحيوية للثيتوزان / بللورزات النانوسليلوز البلوز المؤكسدة \\ عبير محمد عادل' ، أميرة الشافعى' ، منى توفيق الثيمى' ، عاطف عبدالعزيز ابراهيم' و عبدالجواد محمد

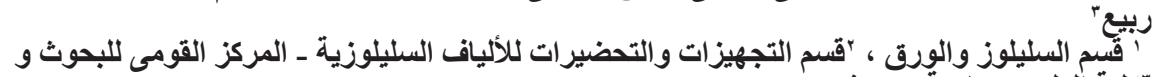

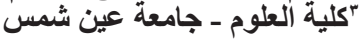

تجذب التعبئة والتغليف الغذائي على أساس المواد القابلة للتحلل الكثير من الاهتمام في الوقت الحاضر . لذلك يعتبر الثشيتوزان

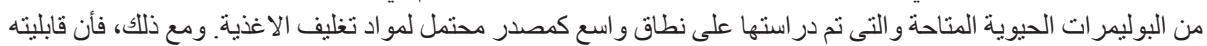

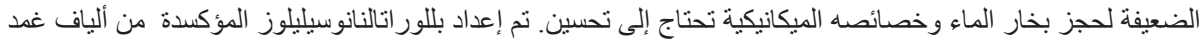

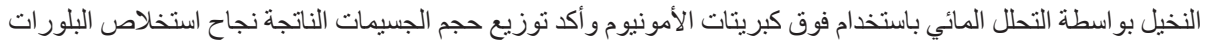

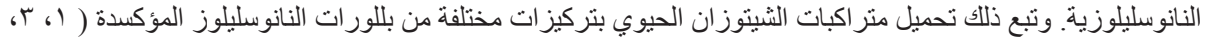

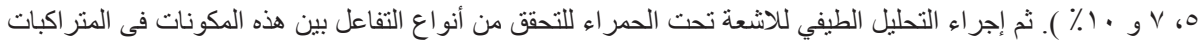

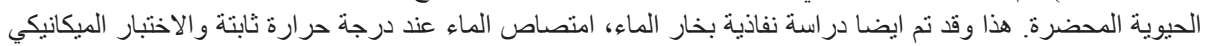

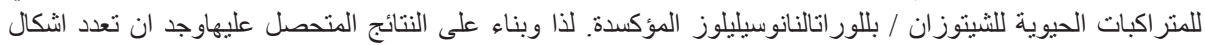

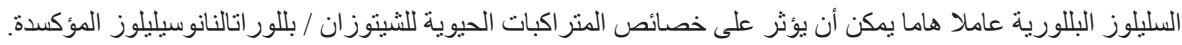

